\title{
Madeleine Guilbert (1910-2006)
}

\section{Marie-Hélène ZYLBERBERG-HOCQUARD}

\section{(2) OpenEdition}

\section{Journals}

Édition électronique

URL : https://journals.openedition.org/clio/2162

DOI : 10.4000/clio.2162

ISSN : 1777-5299

Éditeur

Belin

\section{Édition imprimée}

Date de publication : 1 avril 2007

Pagination : 5-8

ISBN : 978-2-85816-900-9

ISSN : $1252-7017$

Référence électronique

Marie-Hélène ZYLBERBERG-HOCQUARD, « Madeleine Guilbert (1910-2006) », Clio. Histoire, femmes et sociétés [En ligne], 25 | 2007, mis en ligne le 23 septembre 2007, consulté le 23 avril 2022. URL : http:// journals.openedition.org/clio/2162; DOI : https://doi.org/10.4000/clio.2162

Ce document a été généré automatiquement le 23 avril 2022

Tous droits réservés 


\title{
Madeleine Guilbert (1910-2006)
}

\author{
Marie-Hélène ZYLBERBERG-HOCQUARD
}

1 En mars dernier, on apprenait le décès à l'âge de 95 ans, de Madeleine Guilbert. Elle nous a quittés, comme elle le désirait, dans la plus grande discrétion; ce qui ne nous empêche pas de lui rendre hommage. Certes, elle était sociologue et le revendiquait, mais, à la différence de beaucoup de ses collègues, elle a affirmé, à travers ses travaux, l'importance, pour toute recherche, de l'ancrage historique. Surtout, ayant participé à la genèse de la sociologie du travail, elle n'a jamais ignoré les rapports sociaux de sexes. En ces temps lointains, elle était bien la seule, avec Viviane Isambert-Jamati, à le faire.

2 Née avant la Première Guerre Mondiale dans l'Allier, un département profondément républicain, radical voire socialiste, elle était fille d'instituteurs. Elle-même disait de son père, représentatif de son milieu et de sa région : « son anticléricalisme lui servait de ligne politique ». Filles comme garçons, les enfants d'instituteurs étaient encouragés à poursuivre leurs études, elle fréquente donc le lycée de jeunes filles de Montluçon, puis celui de Moulins. Alors qu'entre les deux guerres peu nombreuses sont les filles qui vont au delà du Certificat d'études ou, au mieux, du Brevet Supérieur, et que, souvent, les filles d'instituteurs, à la différence de leurs frères, sont encouragées à présenter le concours de l'Ecole Normale plutôt que le Baccalauréat, elle part faire des études de philosophie à Paris. Un classique destin l'attend, des parents dans l'enseignement primaire, qui n'ont pas de fils, l'ont préparée à devenir professeur. Mais elle tombe malade. La tuberculose touche alors beaucoup d'étudiants. C'est après 1945 grâce aux antibiotiques qu'elle sera définitivement à l'abri des rechutes. Elle passe la guerre à Marseille où son époux, le lexicologue Louis Guilbert, a été nommé enseignant ; ils y participent à la Résistance. A la Libération, elle adhère au Parti Communiste ; avant la guerre, étudiante, elle avait rejoint brièvement les rangs des Jeunesses Socialistes.

3 La maladie, la guerre l'ont éloignée du chemin tout tracé qui devait être le sien; aussi à la Libération saisit-elle l'opportunité qui lui est offerte : elle entre au Ministère du Travail et de la Sécurité Sociale dirigé par Ambroise Croizat. Elle y conduit, à la demande du ministre, des

4 recherches dans le cadre du programme de reconstruction. C'est ainsi qu'elle effectue des enquêtes sur le travail des femmes salariées. En 1946-1947, elle publie, à partir de 
celles-ci, trois articles novateurs dans La Revue Française du Travail, articles d'autant plus originaux qu'elle y fait référence à l'histoire, et esquisse une présentation de la naissance du syndicalisme féminin. Le départ de Croizat entraînant le sien, elle doit abandonner une recherche en cours sur les comités d'entreprise, mais participe à la création de la Revue des Comités d'entreprise de la CGT, et en est, pendant deux ans, secrétaire de rédaction. On peut parler d'une formation proche du terrain, avec une volonté, partagée alors par beaucoup, de voir ses recherches immédiatement utilisables par ceux qui en sont l'objet ; elle restera toujours une militante syndicale avec parfois d'importantes responsabilités, et une volonté d'amener les femmes à l'engagement.

En 1950, elle entre au CNRS, dans le laboratoire de Georges Friedmann ; elle a en effet rédigé un projet sur le travail des femmes qui l'a intéressé. Attachée à l'égalité entre hommes et femmes, ayant trouvé dans ses premières enquêtes plus de questions que de réponses, elle y poursuit, dans une atmosphère novatrice, un chemin non balisé. Par sa présence active, son affirmation qu'un travailleur peut être aussi une travailleuse, elle contribue à ce que la sociologie du travail ne s'enferme pas dans un univers sexuellement neutre, ne néglige pas totalement l'activité féminine, et mette en place quelques concepts spécifiques. Elle reste au CNRS jusqu'en 1969, stagiaire à ses débuts, elle termine maitre de recherches. Certes, elle reconnaissait elle-même que, si ses collègues appréciaient ses recherches sur le travail des femmes, ils y voyaient davantage un ouvrage de dames qu'une pratique de sociologue. Cependant la valeur de ses observations, objectives et chiffrées, son respect des règles scientifiques, auxquelles elle était d'autant plus attachée que son militantisme risquait de la faire taxer de parti pris, lui valent très tôt une reconnaissance universitaire. Elle est d'abord envoyée par Friedmann auprès des ouvrières des Usines Renault; elle élabore un questionnaire, observe les postes de travail, dialogue. Souffrant de son manque de formation, de l'inexistence d'exemples antérieurs, elle élabore ses propres méthodes reposant d'abord sur une parfaite empathie avec son terrain de recherche; des décennies plus tard, elle évoquait encore Maria, une des ouvrières qui lui firent découvrir un milieu que jusque là elle avait ignoré. En 1955, travailler sur les ouvrières à domicile dans la confection parisienne avec Viviane Isambert-Jamati leur permet d'affiner leurs outils, et d'aboutir à la conclusion, inattendue alors : ces femmes qui travaillent à domicile, souvent dans des conditions très dures, le font rarement par obligation familiale, mais parce qu'elles souhaitent correspondre à une certaine image de la femme, encore dominante, en restant au foyer. Madeleine Guilbert peut enfin se lancer dans une thèse sur les fonctions des femmes dans l'industrie, sous la direction de Georges Gurvitch. Elle étudie, sur place, dans les ateliers, suivant à dessein le schéma classique des études de postes (contenu de la tâche, rythme, contraintes, etc...) un important échantillon représentatif de l'emploi des femmes dans les industries des métaux. Elle aboutit à un constat sans équivoque : il existe des spécificités communes aux travaux spécialement attribués aux femmes dans l'industrie (travaux parcellaires, répétitifs, contraignants, accomplis dans des conditions pénibles), spécificités reconnues et acceptées par les intéressées et confirmées par le discours patronal. Lors de sa soutenance, le président, R. Aron, ne trouve pas le sujet très intéressant, n'y voyant qu'une simple enquête commentée; probablement un regard d'une femme sur des femmes, donc négligeable, d'autant que, pour la première fois, un ouvrage scientifique montrait la parenté entre le travail industriel réservé aux femmes et l'activité ménagère. Docteur, elle devient en 1969 professeur de sociologie à Tours, et le reste jusqu'à sa retraite en 1979. Sous la direction d'Ernest Labrousse, elle avait présenté une thèse annexe, devenue elle aussi 
un ouvrage de référence, Les femmes et l'organisation syndicale jusqu'à 1914 ; volonté sans doute de faire des femmes en général, des ouvrières en particulier, des sujets historiques, comme elle en avait fait des sujets sociologiques. Elle est certes loin du concept de genre et plus près, le plus souvent, d'une simple histoire des femmes, cependant sa volonté de ne pas mettre en cause l'unité de la classe ouvrière la conduit à ne pas les étudier isolément. La notion de surexploitation à laquelle elle était attachée peut, étrangement, mener à celle de rapports sociaux de sexes. Pour Madeleine Guilbert, faire découvrir aux femmes leur passé comme la réalité de leur présent, c'était leur donner des armes pour l'action. Elle-même ne s'isole pas dans la recherche, elle est membre du Comité du Travail Féminin depuis sa création en 1965, jusqu'en 1981, responsable de diverses commissions, entre autres de celle sur l'égalité des salaires masculins et féminins ; elle fut membre aussi de la Commission de la main d'oeuvre du $V^{\text {ème }}$ plan. Mieux reconnue, elle peut conduire les premières recherches sur les modes de recrutement de la main d'oeuvre, féminine comme masculine. Elle entreprend, le plus souvent en collaboration avec Nicole Lowit et Joseph Creusen, une série d'études sur les budgets-temps, notamment une étude comparative des budgets-temps hommes/ femmes exerçant une profession. Et, toujours en collaboration, elle réalise la première étude sur les entreprises de travail temporaire alors en plein essor, avec des questions toutes sexuées, et cette conclusion : « ... le travail féminin continue à être considéré comme moins nécessaire, la notion de salaire d'appoint a encore cours et le travail temporaire paraît une solution possible pour les femmes qui l'acceptent plus facilement. »

Une chercheuse féministe ? Elle eût récusé cette affirmation, encore qu'à la fin de sa vie elle admettait être féministe si cela consiste à « penser que la position des femmes est injuste », mais restait méfiante devant les mouvements féministes. Elle a marqué une rupture, ouvert des voies négligées, osé poser des questions totalement occultées, choisi des objets "sans intérêt». Sa sensibilité face à un passé dont l'étude doit précéder toutes recherches pour éclairer le présent, est indéniable dès ses premiers écrits et s'affirme dans sa thèse, avec un long chapitre qui montre sa connaissance des recherches sur le $\mathrm{XVIII}^{\mathrm{e}}$ et $\mathrm{XIX}^{\mathrm{e}}$ siècle tout particulièrement, elle fait donc partie de celles qui ont contribué à donner aux femmes une place dans l'histoire. Son honnêteté intellectuelle et sa grande intelligence font d'elle un modèle toujours actuel pour les historiens comme pour les sociologues. 